\title{
Salvamento artroscópico de menisco lateral secundario a fractura de meseta tibial AO 41B3.1
}

\author{
Arthroscopic salvage of lateral meniscus secondary \\ to tibial plateau fracture AO 41B3.1
}

\author{
Jaime Villalobos $M$, * Rubén García Linage, ${ }^{\ddagger}$ Jonathan R Hernández Molina ${ }^{\S}$
}

\section{Resumen}

Introducción: Las fracturas complejas de los miembros pélvicos incrementan anualmente, dentro de éstas se encuentran las fracturas de meseta tibial. Caso clínico: Masculino de 21 años con fractura compleja de meseta tibial, tratada con osteosíntesis bajo visión artroscópica; como hallazgo artroscópico se observa desinserción meniscal, manejado con ocho suturas meniscales en total, para cuerno anterior y posterior. La fractura tibial tratada con sistema de placas bloqueadas (LCP por sus siglas en inglés) $3.5 \mathrm{~mm}$ e injerto óseo y con evolución a 44 días postquirúrgicos el paciente deambula sin problema con adecuados arcos de movimiento de rodilla. Conclusiones: Las reducciones de fractura de meseta tibial que presentan hundimiento se deben realizar bajo vigilancia artroscópica para observar la congruencia articular de los meniscos y en su caso, efectuar salvamento meniscal.

Palabras clave: Fractura meseta tibial, reducción asistida por artroscopia, sutura meniscal, salvamento meniscal artroscópico

\section{INTRODUCCIÓN}

Las fracturas complejas de los miembros pélvicos se incrementan anualmente, dentro de éstas se encuentran las fracturas de meseta tibial, las cuales pueden resolverse de

* Titular de Curso de Cirugía Articular de Hombro y Rodilla.

₹ Residente de Ortopedia y Traumatología. Facultad Mexicana de Medicina de la Universidad La Salle.

$\S$ Titular adjunto de Curso de Cirugía Articular de Hombro y Rodilla.

Hospital Ángeles Mocel. Ciudad de México, México.

Correspondencia:

Jonathan R Hernández Molina

Correo electrónico: jonyhm@hotmail.com

Aceptado: 11-12-2019.

www.medigraphic.com/actamedica

\section{Abstract}

Introduction: Complex fractures of the pelvic limbs, increase annually, within these, are the fractures of the tibial plateau. Clinical case: 21-year-old male with complex tibial plateau fracture, treated with osteosynthesis under arthroscopic vision, observing as arthroscopic finding, meniscal desinsertion, managed with 8 meniscal sutures in total, for anterior and posterior horn. The tibial fracture was treated with $3.5 \mathrm{~mm}$ locking compression plate (LCP) blocked proximal plate and bone graft, 44 post-surgical days, the patient walks without problem with good knee movement. Conclusions: Tibial plateau fracture reductions when there's articular involvement, should be made under arthroscopic surveillance to observe the joint congruence of the meniscus and, where appropriate, perform meniscal rescue.

Keywords: Tibial plateau fracture, arthroscopically assisted reduction, meniscal suture, arthroscopic meniscal salvation.

forma quirúrgica o conservadora. La tasa de satisfacción es de $44 \%$, lo cual implica alto índice de complicaciones. ${ }^{1}$

Las lesiones meniscales pueden ser tratadas de forma conservadora o quirúrgica, en caso de requerir cirugía debe optarse por la mínima invasión, pudiendo resultar en una meniscectomía parcial o meniscoplastia; la meniscectomía total ya no se utiliza. ${ }^{2}$

Las fracturas de meseta tibial intraarticulares representan un reto por su complejidad, siendo prioritaria la reducción anatómica directa, sea ésta abierta o vía artroscópica. $M$. Le Baron y colaboradores reportan no haber encontrado diferencia significativa entre ambos métodos. ${ }^{3}$

Es de recalcar que las lesiones a nivel articular con frecuencia se asocian a lesiones cartilaginosas, las cuales pueden presentarse en el evento agudo, o ser consecuencia de evento artrítico crónico, en el cual el tratamiento es más complejo y no tienen tan buenos resultados como en lesiones agudas. Aproximadamente 
$45 \%$ de los pacientes quedan con alguna secuela articular. En casos extremos se llega a optar por realizar un reemplazo total de rodilla en el evento agudo, situación infrecuente incluso en centros de referencia mundiales de estas lesiones. Los factores que van a determinar los tipos de lesiones y los lugares que van a ser más afectados en una osteoartritis de rodilla son: el sitio de carga, la inestabilidad y el eje axial. ${ }^{4}$

La reducción asistida por artroscopia se ha vuelto popular en los últimos años, la gran ventaja es reducir bajo visión directa sin necesidad de dañar las inserciones meniscales. ${ }^{5}$
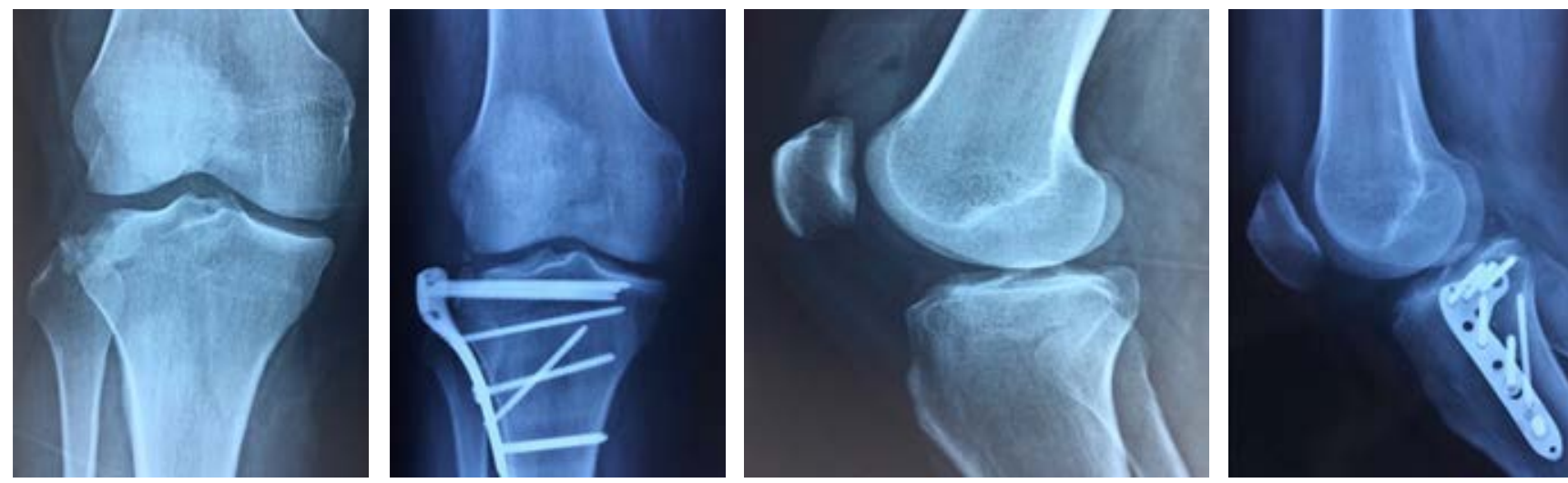

Figura 1: Fractura de meseta tibial con hundimiento lateral/osteosíntesis con adecuada reducción y colocación de placa anatómica.
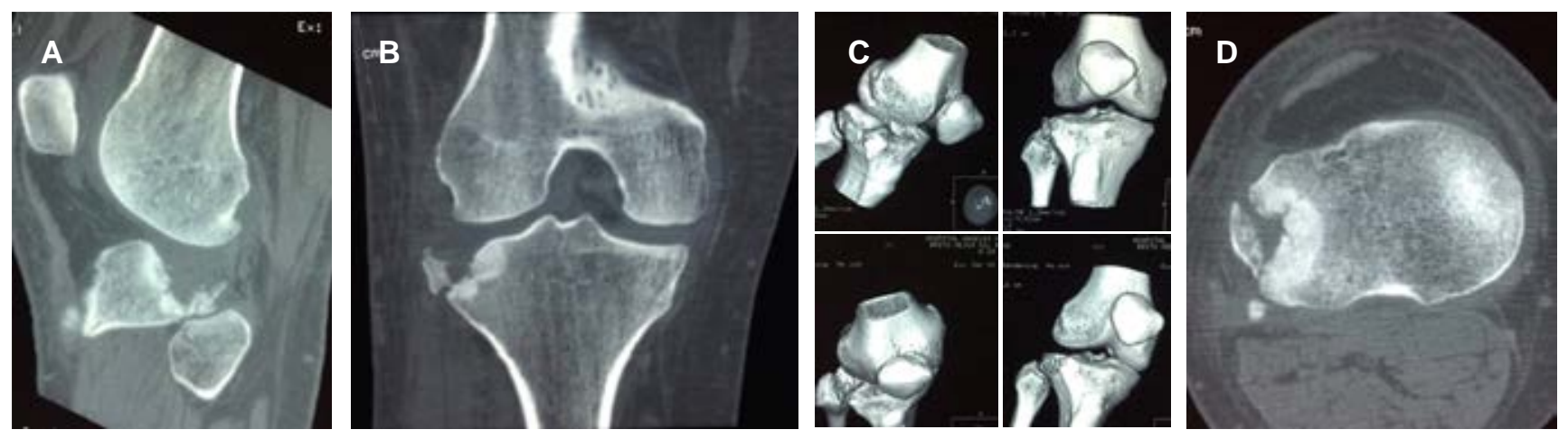

Figura 2: Tomografía de rodilla que evidencia el hundimiento articular. (A) Corte sagital. (B) Corte coronal. (C) Reconstrucción en 3D. (D) Corte axial.

Figura 3:

Imagen artroscópica que evidencia la desinserción meniscal y la colocación de

suturas.
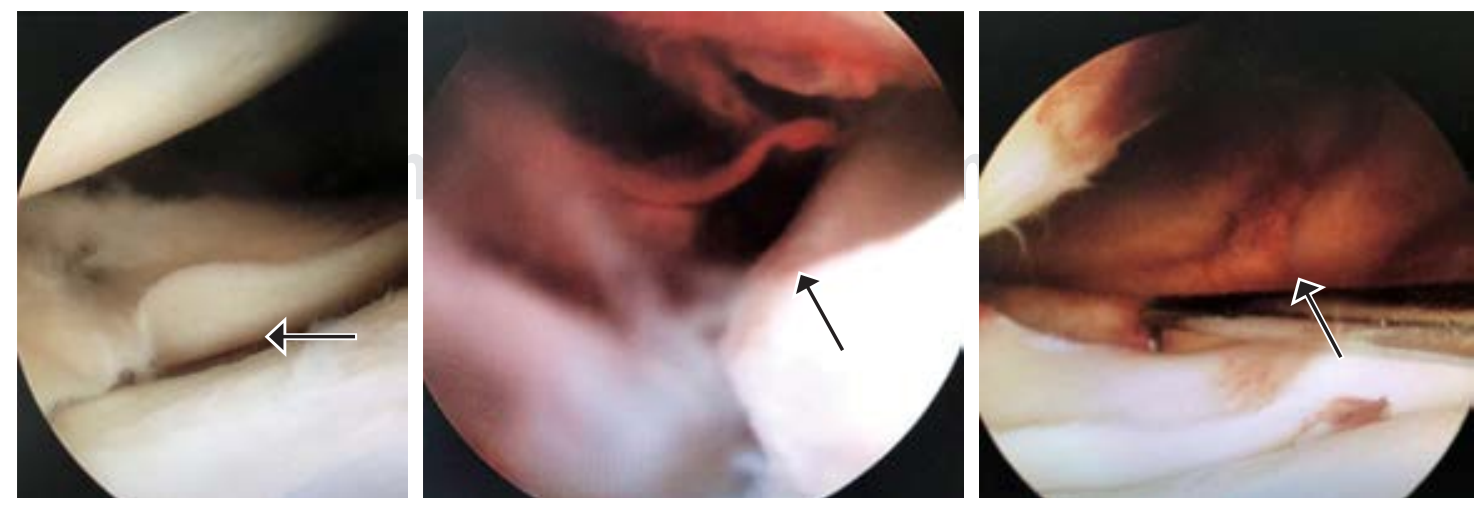


\section{DESCRIPCIÓN DEL CASO}

Masculino de 21 años sin antecedentes de importancia inicia padecimiento actual al realizar actividad física, recibe contusión directa en rodilla derecha que ocasionó valgo forzado. Refiere dolor localizado en meseta tibial lateral con incapacidad para deambular, y arcos de movimiento disminuidos por dolor con predominio a la flexión.

\section{Exploración física}

Miembro pélvico derecho con arcos de movimiento de rodilla derecha limitados por dolor, presenta hematoma de $2 \times 3 \mathrm{~cm}$ en región lateral de rodilla, y aumento de volumen con incremento de temperatura, cajones y bostezos no valorables. Pruebas de McMurray, Steinmann, Thessaly y Apley no valorables, cepillo negativo, escape negativo, neurovascular distal íntegro. Miotomos en escala de Daniels: de L2a S1 en calificación 5/5 bilateral. Dermatomos íntegros de L2-S1. Reflejos rotuliano y aquíleo en ++ bilateral.

Con diagnóstico clínico de fractura de meseta tibial se solicita: radiografías AP y lateral de rodilla derecha, tomografía computarizada y resonancia magnética: en las radiografías se observa pérdida de continuidad ósea de meseta tibial lateral con hundimiento y multifragmentación y lateralización de patela; la tomografía mostró fractura desplazada de meseta tibial con compromiso articular con multifragmentación articular; en resonancia magnética nuclear (RMN) rodilla derecha: concluyendo los diagnósticos de: a) fractura meseta tibial derecha AO 41B3.1, Schatzker III; b) fractura cabeza de peroné derecho, c) lesión menisco lateral rodilla derecha y d) lesión parcial del ligamento colateral lateral derecho.

Se realizó artroscopia de rodilla que evidenció hundimiento de meseta tibial con desprendimiento de cuerno anterior hasta cuerno posterior de menisco lateral, además de lesión grado I de menisco medial, y lesión condral grado I Outerbridge en región lateral de patela. Se realizó abordaje lateral de tibia, se redujo la fractura con levantamiento de platillo tibial y se colocó $10 \mathrm{~cm}^{3}$ de injerto óseo en defecto, se corroboró adecuado levantamiento vía artroscópica, se observó adecuada congruencia articular, y se colocó placa proximal bloqueada de tibia $3.5 \mathrm{~mm}$ tipo Depuy Synthes.

Se introdujo nuevamente el artroscopio, reduciendo el menisco lateral fijándolo con ocho suturas, cuatro Suture Lasso para cuerno anterior con técnica de afuera hacia adentro y cuatro Fast-Fix para cuerno posterior con técnica de adentro hacia adentro.

En su evolución a los 44 días de cirugía se encontró marcha normal sin apoyo, arcos de movimiento completos de rodilla, datos meniscales de rodilla negativos y sin datos de lesión de ligamento cruzado anterior ni ligamentos colaterales. Fuerza de cuádriceps 4/5 en escala de Daniels, rodilla estable.

\section{DISCUSIÓN}

Fisiopatología: en las fracturas por hundimiento de la meseta tibial, los meniscos pueden perder parte de su inserción menisco-capsular y llegar a fracturarse. Con estas lesiones, la rodilla pierde el soporte y existe el riesgo de gonartrosis temprana.

El riesgo que representa una lesión tanto meniscal como de ligamento cruzado anterior en pacientes jóvenes, como se ha mencionado anteriormente, es la evolución a osteoartrosis, la cual puede tener secuelas importantes como la limitación articular que condiciona dificultad para llevar a cabo tareas diarias y más aún si el paciente es atleta o hace ejercicio de forma cotidiana.

Existe la corriente de no realizar ningún tipo de reparación en el primer abordaje donde se efectúa la osteosíntesis, ya que prolonga el tiempo de cirugía y se considera que los riesgos no son importantes a largo plazo. La mayoría de los pacientes con fracturas Schatzker 5 y 6 no regresarán al nivel de actividad física que tenían previamente. ${ }^{6}$

En las fracturas por depresión de la meseta tibial existen componentes de tejidos blandos que tienen gran importancia en el pronóstico del paciente a largo plazo. Al ser los meniscos los estabilizadores principales de la rodilla deben buscarse lesiones tanto en cuernos y en cuerpo, repararlas al momento de la artroscopia y finalmente corroborar adecuada congruencia articular.

Dentro de las fracturas de meseta tibial, Y. Warschawski y colaboradores indican que las clasificadas en Schatzker tipo $\mathrm{V}$ y VI es difícil que regresen a la normalidad, pues con frecuencia dejan secuelas en los arcos de movimiento, las clasificadas por debajo de éstas muestran mejor reha-
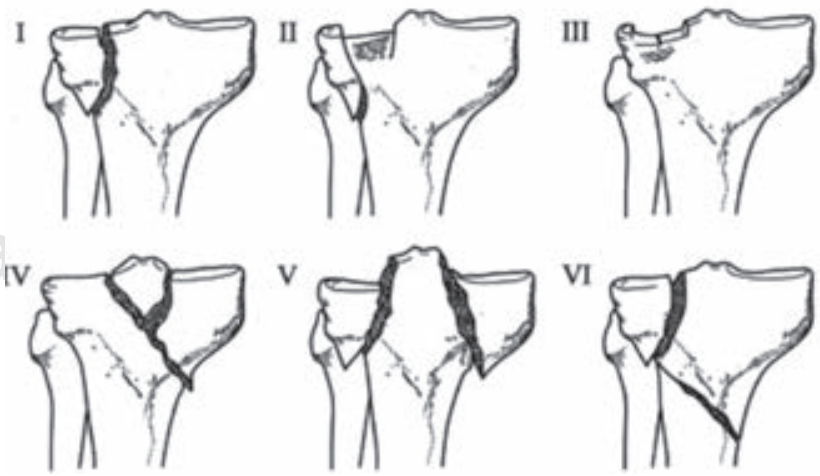

Figura 4: Clasificación de Schatzker para fracturas de meseta tibial. 
bilitación, siendo más factible que recuperen la función de la rodilla. ${ }^{6}$

En casos como el presentado los programas de fisioterapia y rehabilitación resultan indispensables para completar arcos de movimiento y restaurar la función.

Existen algunas fracturas de meseta tibial que pueden pasar desapercibidas en la radiografía simple de rodilla, por lo cual se sugiere realizar de tomografía en la que se pueda apreciar si existe trayecto articular o no. Para el diagnóstico de lesión de tejidos blandos la resonancia magnética es la elección, ya que permite valorar la situación intraarticular y especialmente auxilia en la planificación de la cirugía. ${ }^{7}$

Las complicaciones de las fracturas de alta energía en la meseta tibial, cuyo tratamiento no es el adecuado son: a) pérdida de la reducción; b) incremento del riesgo de trombosis venosa profunda; c) infecciones, en caso de las tempranas, y las tardías, no-unión, desgaste o ruptura del material de osteosíntesis, d) rigidez de rodilla o anquilosis por fibrosis y e) osteoartritis postraumática a mediano plazo. La disminución del movimiento de la rodilla o la pérdida completa de los grados de movimiento es común. No sólo las lesiones óseas desempeñan un papel, es importante entender que el aparato extensor y flexor se inmoviliza, por lo cual se pierde momentáneamente la función, de igual forma existe la artrofibrosis que es uno de los factores que influye en la disminución de la movilidad articular. Recalcar siempre la importancia de realizar rehabilitación física oportuna, ya sea el caso del tratamiento quirúrgico o bien el tratamiento conservador. Se ha incrementado el nivel de actividad física a menor edad y esto ha aumentado la incidencia de estas lesiones, se debe optar por un tratamiento eficaz y sin retraso para permitir la adecuada función articular.

\section{CONCLUSIONES}

Cuando hay hundimiento las reducciones de fractura de meseta tibial se deben realizar bajo vigilancia artroscópica para observar la congruencia articular de los meniscos y en su caso, efectuar salvamento meniscal buscando, siempre que sea posible, la preservación meniscal con reparación y evitar la meniscectomía especialmente en pacientes jóvenes.

\section{REFERENCIAS}

1. Cuéllar-Avaroma A, King-Martínez A, Hernández-Salgado A, TorresGonzález R. Complicaciones en las fracturas complejas de la meseta tibial y factores asociados. Cir Ciruj. 2006; 74 (5): 351-357.

2. Pujol N, Boisrenoult P, Beaufils P. Lesiones traumáticas de los meniscos de la rodilla. EMC - Aparato Locomotor. 2014; 47 (2): 1-10.

3. Elabjer E, Benčić I, Ćuti T, Cerovečki T, Ćurić S, Vidović D. Tibial plateau fracture management: arthroscopically-assisted versus ORIF procedure - clinical and radiological comparison. Injury. 2017; 48 Suppl 5: S61-S64.

4. Aurich M, Koenig V, Hofmann G. Comminuted intraarticular fractures of the tibial plateau lead to posttraumatic osteoarthritis of the knee: Current treatment review. Asian J Surg. 2018; 41 (2): 99-105.

5. Hung SS, Chao EK, Chan YS, Yuan LJ, Chung PC, Chen CY et al. Arthroscopically assisted osteosynthesis for tibial plateau fractures. J Trauma. 2003; 54 (2): 356-363.

6. Warschawski Y, Elbaz A, Segal G, Norman D, Haim A, Jacov E et al. Gait characteristics and quality of life perception of patients following tibial plateau fracture. Arch Orthop Trauma Surg. 2015; 135 (11): 1541-1546.

7. Carredano X, Valderrama J, Marín F, Valderrama I, Espinoza G. Complicaciones en fracturas de platillos tibiales de alta energía. Rev Chil Ortop Traumatol. 2016; 57 (3): 70-75. 